\title{
Dissipative and Fluctuating Dynamical Description of Nuclear Reactions
}

\author{
V. de la Mota, F. Sébille, and C. Bonilla \\ Subatech, 4 rue A. Kastler, BP 2072244307 Nantes cedex 03, France
}

Received on 11 September, 2003

\begin{abstract}
We address the description of nuclear dynamics at intermediate energies within the DYWAN model. New fluctuation-dissipation aspects are discussed and confronted with experimental results in nucleus-nucleus and in nucleon-nucleus reactions. We show that the implemented treatment of fluctuations provides with a convenient tool to investigate the main characteristics of particle and light clusters emission in the intermediate energy range.
\end{abstract}

\section{Introduction}

In this work we present an approach for the description of nuclear dynamics: the dywan model which has been developped in the spirit of the projection methods of statistical physics (PM) [1] and on the wavelet theory (WT) [2]. After a brief description in Sec. 2 of our approach, we present in Sec. 3 two kind of calculations, one of them concerns dissipation aspects in heavy-ion collisions at intermediate energies, the other, nucleon and light cluster emission in nucleon-nucleus reactions in the same energy range.

\section{Background}

PM are at the origin of the physical formulation, which consists essentially in the extended time-dependent HartreeFock (ETDHF) equation,

$$
i \dot{\rho}=[h(\rho), \rho]+i I(\rho)
$$

derived with the assumption that only one-body observables are relevant for the description of the system. Here $\rho$ is the one-body density matrix, $h(\rho)$ is the one-body hamiltonian and I the collision kernel.

The WT provides the resolution schemes for the above equation of motion. Wavelets are nonstationnary bases. A family of wavelets is obtained by translating and expanding the generating function, called mother (or analysing) wavelet:

$$
\Psi_{a, b}(x)=\frac{1}{\sqrt{a}} \Psi\left(\frac{x-b}{a}\right)
$$

where $a$ and $b$ are, respectively, the compression and translation parameters. This property permits to perform an analysis of the signal at different scales.

Expanding in wavelets the single-particle (s.p.) wavefunctions it can be shown, from Eq. (1), that the dynamics is generated by the evolution of the moving wavelets in the mean-field between two transitions. This evolution is given in terms of a system of coupled equations of motion [3] for the centroids and the widths of wavelets. Since the orthogonality of wavelets is conserved in time, Pauli blocking is ensured all over the dynamical evolution.

In order to go beyond the one-body description, in a first time, we look for the least biased many-body wavefunction, compatible with the information contained in our ETDHF equation. Indeed, due to the orthonormality properties of s.p. wave-functions and wavelets, the most general $\mathrm{N}$-body wave-function takes the form of a linear combination of Slater determinant of wavelets. The correpondig Nbody density operator can be written, in the random phase approximation, as a statistical mixture of N-body Slater determinants of non-stationary wavelets as follows:

$$
D^{N}(t)=\sum_{K}\left|a_{K}(t)\right|^{2} \sum_{M, M^{\prime}} b_{M} b_{M^{\prime}}^{*}\left|\Theta_{M}><\Theta_{M^{\prime}}\right|
$$

Here, $\mid \Theta_{M}>$ is a Slater determinant of wavelets, $b_{M}=$ $\operatorname{det}\left(c_{\lambda}^{\mathrm{i}}\right)$ and the $c_{\lambda}^{i}$ are the coefficients of the decomposition of s.p. wave-functions on the wavelets basis. The subsequent evolution, at the N-body level, is given by $D^{N}(t)$, which is treated by means of a Montecarlo procedure, in consistency with the simulation of the master equation. This procedure introduces some degree of stochasticity in the treatment of $\mathrm{N}$-body information, in a similar way as stochastic models.

\section{Results}

In the following, the local Zamick force [4] and the free nucleon-nucleon cross-section $\sigma_{n n}$ have been implemented in numerical calculations.

In Fig. 1 the recoil velocity of the binary system, normalized to the $\mathrm{CM}$ velocity, is represented in the $\mathrm{Ar}+\mathrm{Ag}$ reaction. The data is in good agreement with the two dynamical models, in which the impact parameter range has been selected in order to eliminate fusion events. 

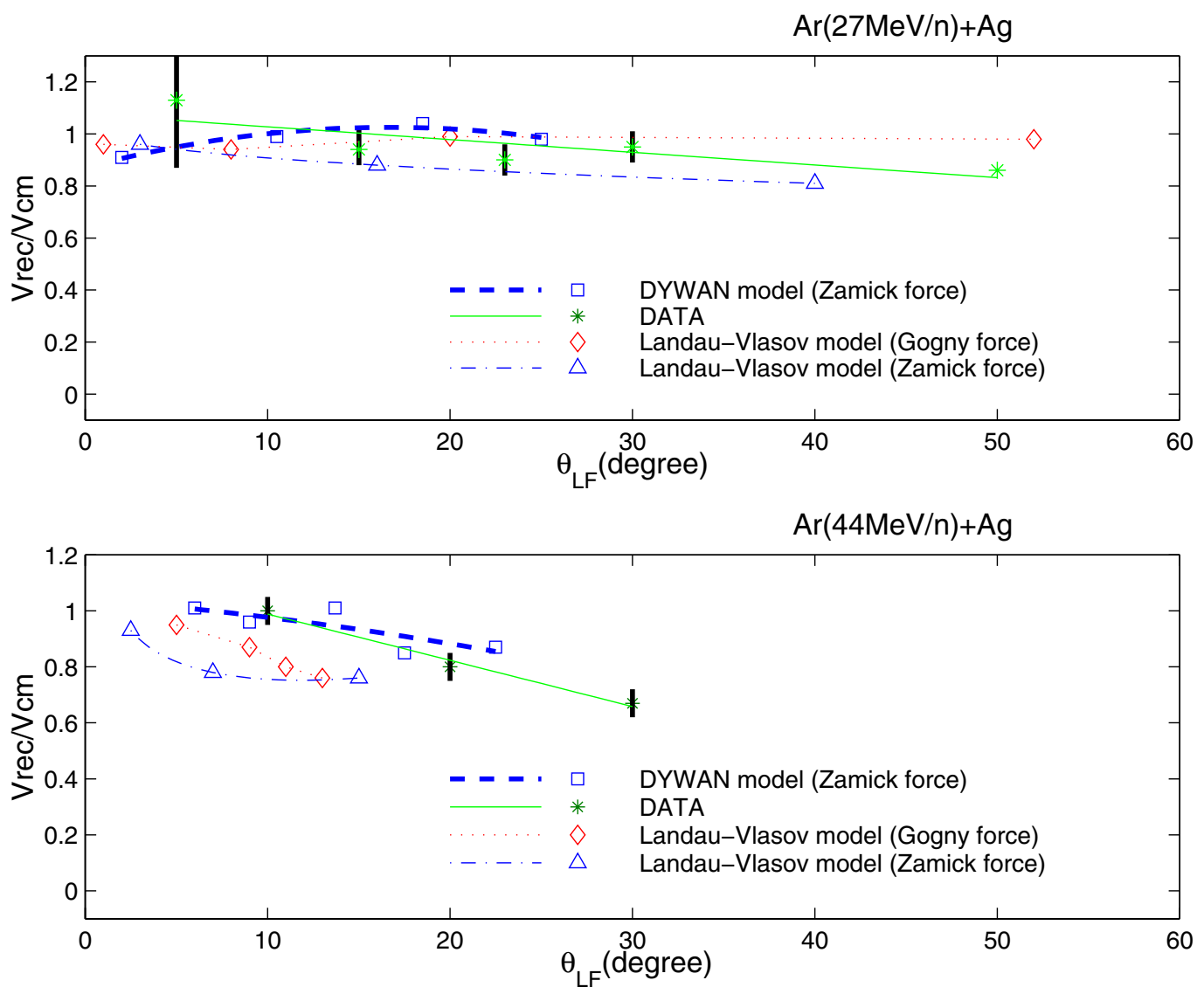

Figure 1. Normalized recoil velocity of heavy fragments as a function of the light fragment angle in $\mathrm{Ar}+\mathrm{Ag}$ reactions at $27 \mathrm{MeV} / \mathrm{n}$ (top) and $44 \mathrm{MeV} / \mathrm{n}$ (bottom).

Nevertheless, we observe the following main differences:

- the semi-classical (s.c.) LV results are sensitive to the effective force only at the highest incident energy, where for the non-local force, relative velocities are higher and as a consequence the stopping power is less important.

- dywan results are in better agreement with the data, the reason being the spreading of the s.p. wave-functions which takes place in the overlap region, and which will contribute to increase the deflection angle of the light partner.

From these results we do not have to conclude that the dywan model, with the Zamick force, gives the right description of the reaction dynamics. They underline the importance of quantum effects, which need to be taken into account before undertaking the study of more sophisticated effects like in-medium and surface effects.

In Ref [5] proton double-differential cross-sections in $\mathrm{n}+{ }^{208} \mathrm{~Pb}$ reaction at $96 \mathrm{MeV} / \mathrm{n}$ have been calculated for some selected angles. The results are globally correctly reproduced. For energies between 20 and $100 \mathrm{MeV}$ out-ofequilibrium effects are strong: an important anisotropy appears in spectra. These results suggest that the role of the mean-field at these energies cannot be neglected.

In Fig. 2 the importance of self-consistency is investigated in proton double differential cross-sections for the same reaction as above. On the left is the usual self- consistent calculation whereas on the right a calculation with a frozen mean-field is reported. We observe that the role of the self-consistent field is essential to keep a better agreement with the experimental results. The results obtained with a constant (initial) mean-field underestimate the data.

\section{Conclusions}

The DYWAN model has been developped to describe HIC and nucleon induced reactions at intermediate energies. The motivation was the understanding of the decay mechanisms in those reactions and to learn about the properties of very far from equilibrium nuclear matter (stability limits, fragment formation, vaporization, ...).

Comparing with the experimental data, even with a local force and without in-medium corrections, we have shown that the model describes correctly the dissipative mean behavior, improving s.c. calculations. This fact is directly connected with the time evolution of the wavelet basis. We have also shown that nucleon yields in nucleon induced reactions are in good agreement with the data. In particular, the contribution of peripheral events to the spectra is shown to be dominant. Since these spectra can be sensitive to surface effects, they can be utilized as a probe of these kind of effects. 


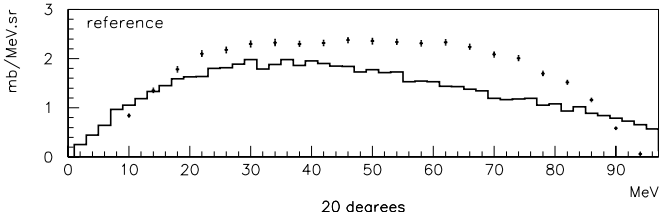

20 degrees

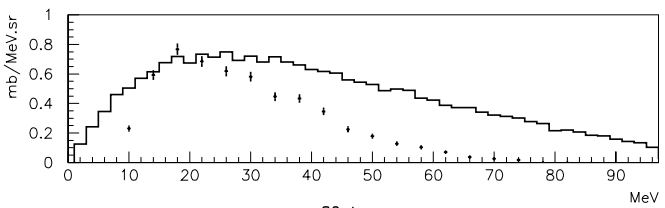

80 degrees

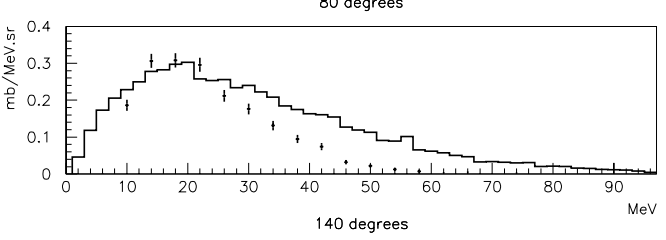

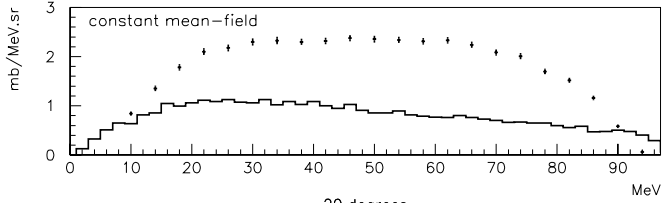

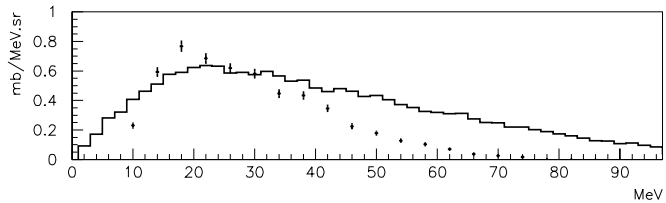

80 degrees

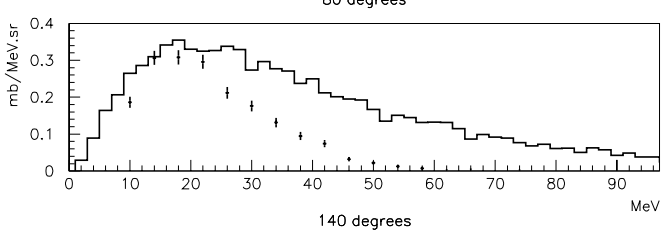

Figure 2. Proton double differential cross section for ${ }^{208} \mathrm{~Pb}(n, x p)$ reaction at $96 \mathrm{MeV}$. The left panel is the reference self-consistent calculation, in the right is a simulation without self-consistency. Experimental data is represented by crosses.

The dywan model can then make qualitative and quantitative predictions in nuclear reactions at intermediate energies, and this gives the possibility to investigate different dynamical aspects, as the role of the self-consistency in nucleon-nucleus collisions. This fact constitutes an important constraint for the concerned models. Finally, our results underline the fact that quantum structure has to be taken into account, in order to investigate properly more refined physical aspects (as those related with surface and in-medium effects). This work is in progress.

\section{References}

[1] R. Balian, Y. Alhassid, and H. Reinhardt, Phys. Rep 1311 (1986).

[2] A. Grossmann, J. Morlet, T. Paul, Ann. Inst. Poincaré 45, 293 (1986).

[3] B. Jouault, F. Sébille, and V. de la Mota, Nucl. Phys. A 628, 119 (1998).

[4] L. Zamick, Phys. Lett. B 45, 313 (1973).

[5] C. Bonilla et al., Proceeding of the $10^{\text {th }}$ Int. Conf. on Nucl. React. Mechanisms, Varenna (Italy) june 2003. 